\title{
Defibrillator twiddler's syndrome: a rare cause of implantable cardioverter-defibrillator failure
}

\author{
A Navone, A Picone, $\mathrm{K}$ Boahene
}

Division of Cardiology, State University of New York, Health Science Center at Syracuse, Syracuse, New York, USA

A Navone

$\mathrm{K}$ Boahene

Division of Cardiac

Surgery, State

University of New

York, Health Science

Center at Syracuse,

Syracuse, New York,

USA

A Picone

Correspondence to:

Dr A Navone,

Cardiovascular Division,

State University of New

York, Health Science Center

at Syracuse College of

Medicine, 750 East Adams

Street, Syracuse, New York

13210, USA.

Accepted for publication 25 June 1996

(A) Pre-discharge chest $x$ ray confirming position of ICD leads. Black arrow indicates right ventricular lead and white arrows indicate superior vena cava and innominate vein leads. (B) Chest $x$ ray showing the change in lead position. See text for details.
Manipulation of the implantable cardioverterdefibrillator (ICD) generator may cause lead fracture, lead dislodgement, and insulation breaks. All these defects can result in spurious shocks being given. In this report we present a case of defibrillator twiddler's syndrome that resulted in the near inability to terminate ventricular fibrillation.

The patient was a 57 year old man with a cardiomyopathy who had had ICD implantation a year before for ventricular fibrillation. A non-thoracotomy lead system consisting of two superior vena cava coils and a right ventricular screw-in lead were used. The generator was implanted subcutaneously over the rectus fascia below the left subcostal area. The postoperative course was uneventful and a chest $x$ ray before hospital discharge revealed no changes in lead position (fig 1A). A subcutaneous patch was subsequently explanted owing to an infection. Repeat testing showed that defibrillation thresholds were adequate when the transvenous lead system alone was used.

One year later symptoms of congestive heart failure developed. During treatment with vesnarinone he developed ventricular fibrillation

A

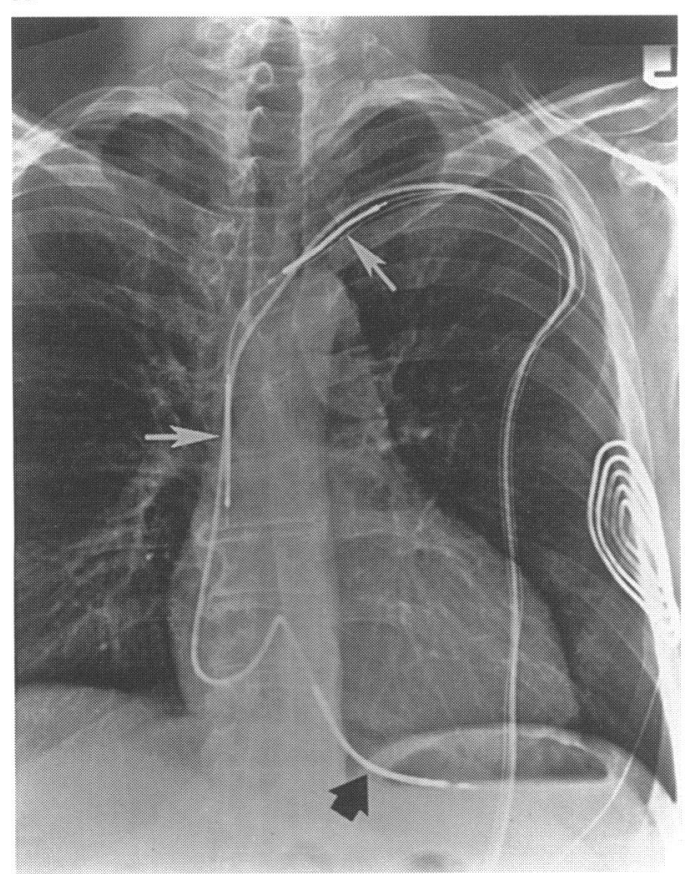

which could not be terminated until a third 34 $\mathrm{J}$ shock from his ICD was delivered. Chest $x$ ray showed that the lead in the innominate vein had migrated out of the vasculature and into the subcutaneous tissue, and the lead at the junction of the superior vena cava and right atrium (RA) was now in the innominate vein. The right ventricular lead had developed a loop within the RA (fig 1B). Surgical exploration of the pulse generator pocket confirmed the fluoroscopic findings of twisted and coiled defibrillator leads.

This case illustrates a rare, but potentially fatal consequence of patient manipulation of an ICD generator. Defibrillator twiddler's syndrome was first reported in $1984 .{ }^{1}$ Like pacemaker twiddler's syndrome, described by Bayless $e t a l{ }^{2}$ the mechanism is thought to be a capstan effect produced by rotating the pulse generator in a capacious pulse generator pocket. Rotation of the pulse generator results in traction on the leads which can eventually dislodge or fracture the lead.

Although most patients who present with pacemaker or ICD twiddler's syndrome deny conscious manipulation of the pulse generator, they often have local discomfort or a sensation

$B$

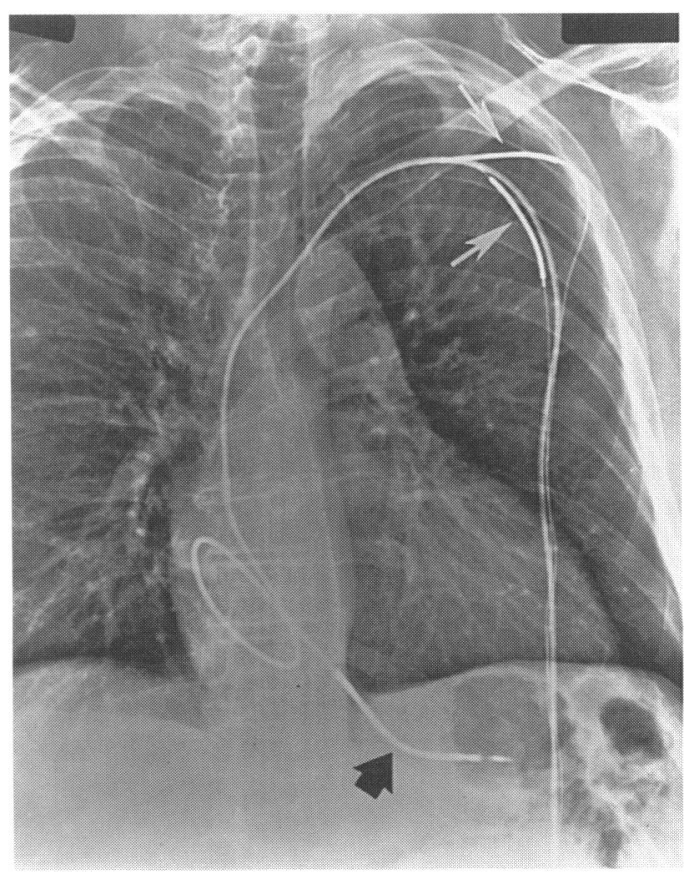


that the generator is moving or flipping around within the subcutaneous pocket. The location of most ICD generators may promote subconscious manipulation. If the generator is placed too high in the abdomen, the patient may push the generator away from the ribs, inadvertently rotating it each time. A report of pacemaker twiddler's syndrome in a $\operatorname{dog}^{3}$ and a recent report of three cases of "spontaneous" coiling of pacemaker leads ${ }^{4}$ raise the possibility that manipulation may not be responsible in some instances. The density of the generator may contribute to this process. ${ }^{5}$ There is no correlation with the type of ICD generator used in the various reported cases of ICD twiddler's syndrome. The eventual development of smaller, lighter, and less dense ICD pulse generators may help resolve some of these difficulties.

This is one of the first cases of ICD twiddler's syndrome to be described in a patient with a completely transvenous, non-thoracotomy lead (NTL) system. In our case, the RV lead was a screw-in type, and did not become dislodged from the ventricle, but the traction on the lead resulted in a loop being formed in the right atrium. The freely floating leads in the superior vena cava and innominate vein migrated out of position despite being secured appropriately at the site of venous access. It is well known that slight changes in lead position, by altering the defibrillation vector, can dramatically affect the defibrillation threshold. Because of these observations, twiddling may be more likely to lead to lack of device efficacy in NTL systems than in thoracotomy systems. It also seems likely that even if twiddling is less common in NTL systems, mortality from this syndrome is likely to be higher if the same $10 \mathrm{~J}$ safety margin for defibrillation threshold is used as the standard for implanting NTL systems. Routine testing of NTL ICDs seems to be warranted in all patients in whom $x$ ray examination shows slight changes in lead position, because there are as yet no known effective techniques to prevent the syndrome.

1 Veltri E, Mower MM, Reid PR. Twiddler's syndrome: new twist. PACE 1984;7:1004-9.

2 Bayless CE, Beanlands DS, Baird RJ. The pacemaker-twiddler's syndrome: a new complication of implantable ransvenous pacemakers. Can Med Ass $\mathcal{F}$ 1968;99:371.

3 Been M, Darke PGG. Pacemaker twiddler; A twist in the tail? BrMed $\mathcal{F}$ 1988;297:1642-3.

4 Gialafos J, Siamas G, Kandicas J, Gatzoulis K, Toutouzas P. Spontaneous twisting of an implantable pacemake electrode in three cases. PACE 1994;18:(Part 1) 1069-71.

5 Furman S. Defibrillator twiddler's syndrome. Ann Thorac Surg 1995;59:544-6. 